\title{
Erratum: Lock-in transition of charge density waves in quasi-one-dimensional conductors: Reinterpretation of McMillan's theory [Phys. Rev. B 97, 115432 (2018)]
}

\author{
Katsuhiko Inagaki and Satoshi Tanda
}

(Received 20 May 2019; published 5 June 2019)

DOI: 10.1103/PhysRevB.99.249901

In our article, we have studied the lock-in transition of charge density waves (CDWs) in quasi-one-dimensional conductors. McMillan's [1] theory was applied to understand various behaviors of the transition. McMillan's [1] free energy has the following form:

$$
F_{1}=F^{0} \int d^{2} s\left[-|\phi|^{2}-\beta Y \operatorname{Re}\left(\phi^{3}\right)+\frac{1}{2}|\phi|^{4}+\beta|\vec{\nabla} \phi+i \phi|^{2}+\gamma\left|-\vec{q}_{1} \times \vec{\nabla} \phi\right|^{2}\right],
$$

where $\phi$ is a phase of the CDW defined as $\psi=\psi_{0} e^{i K \cdot r / 3} \phi(r)$ and $\psi$ is a complex order parameter. We solved it by substituting $\psi=\psi_{0} e^{i K \cdot r / 4} \phi(r)$ for the order parameter and obtained a result similar to Eq. (1); however, it lacks the $\beta Y \operatorname{Re}\left(\phi^{3}\right)$ term because the umklapp term becomes fourth order in this case, namely, proportional to $|\phi|^{4}$. We insisted that the umklapp term gives no energy gain if a phase modulation $\phi=e^{-i \theta(x)}$ alone is considered as in McMillan [1]. However, our calculation implicitly assumed that $\phi$ was not complex, which led us a wrong result. By taking into account it being complex, the following terms are obtained from fourth-order umklapp processes, $\frac{1}{8}\left(\phi^{2}-\phi^{* 2}\right)^{2}+\frac{1}{2}|\phi|^{4}$ where the first term was missed in our article. The new term cancels out if the order parameter is real, namely, $\phi=\phi^{*}$. As shown in Eq. (1), such a cancellation happens to the imaginary part of the order parameter in his derivation of the $\beta Y \operatorname{Re}\left(\phi^{3}\right)$ term.

As for the phase modulation $\phi=e^{-i \theta(x)}$ from which a periodical function $\frac{1}{4}(\cos 4 \theta-1)$ is derived. Hence, we correct the description to:

"the even-number umklapp term gives no energy gain if a phase modulation $\phi=e^{-i \theta(x)}$ alone is considered unless the order parameter is complex."

This correction does not affect other statements of our paper.

[1] W. L. McMillan, Phys. Rev. B 14, 1496 (1976). 